\title{
High Five Model: Los factores Altos están Asociados con Bajo Riesgo de Enfermedades Médicas, Mentales y de Personalidad
}

\author{
Alejandro Castro Solano ${ }^{1}$ y Alejandro César Cosentino ${ }^{2}$
}

\begin{abstract}
RESUMEN
El Modelo de los Cinco Altos (MCA; High Five Model, HFM, por sus siglas en inglés) establece cinco rasgos positivos de la personalidad: erudición, paz, jovialidad, honestidad, y tenacidad, llamados factores altos. Un estudio previo había mostrado la validez convergente, discriminante y estructural del MCA. Este estudio tuvo por objetivo corroborar que el MCA está asociado positivamente a variables relacionadas con la salud física y mental de los individuos. Se realizó este estudio con una muestra de participantes de población general no consultante. Específicamente, se estudió la asociación entre los factores del MCA y el modelo de Salud Mental Positiva, el bajo riesgo de enfermedad médica, la ausencia de sintomas psicopatológicos y de rasgos asociados con la personalidad psicopatológica. Para tal fin, se realizaron dos análisis de correspondencia múltiples que estudiaron las relaciones entre los factores del MCA con, por una parte, la Salud Mental Positiva y el bajo riesgo de enfermedad médica, y, por otra parte, indicadores de trastorno mental o de personalidad. Los resultados mostraron que, en términos generales, puntuaciones elevadas en los factores del MCA están asociadas a indicadores de salud y, contrariamente, puntuaciones bajas en esos factores se asocian a indicadores de enfermedad. Estos resultados aportan a la validez de constructo del MCA.

Palabras clave: rasgos de personalidad, bienestar, psicopatologia, trastornos de la personalidad, diagnóstico médico, validez de la prueba
\end{abstract}

\section{High Five Model: The High Factors are Associated with Low-Risk of Medical, Mental and Personality Diseases}

\begin{abstract}
The High Five Model (HFM; Modelo de los Cinco Altos, MCA, in Spanish) establishes five positive traits of personality: erudition, peace, joviality, honesty, and tenacity, called high factors. A previous study had shown the convergent, discriminating, and structural validity of the HFM. This study aimed to corroborate that the HFM is positively associated with variables related to the physical and mental health of individuals. This study was carried out with a sample of participants from the non-consulting general population. Specifically, the associations between the HFM factors with the Positive Mental Health model, the low risk of medical disease, the absence of psychopathological symptoms and psychopathological personality traits were studied. To that end, two multiple correspondence analyzes were carried out to study the relationships between HFM factors with, on the one hand, Positive Mental Health and the low risk of medical illness, and, on the other hand, indicators of mental disorder or personality. The results showed that, in
\end{abstract}

\footnotetext{
${ }^{1}$ Consejo Nacional de Investigaciones Científicas y Técnicas (CONICET); Departamento de Psicologia, Facultad de Ciencias Sociales, Universidad de Palermo, Argentina; alejandro.castrosolano@gmail.com ${ }^{2}$ Departamento de Psicologia, Facultad de Ciencias Sociales, Universidad de Palermo, Argentina.
}

El presente proyecto fue realizado mediante los subsidios PIP CONICET 11220150100381CO "Hacia una aproximación émica de la psicología positiva. Los rasgos positivos como predictores del funcionamiento óptimo" y con subsidio UBACyT, 20020150100037BA "La evaluación de los rasgos de personalidad positivos. Su relación con los rasgos de personalidad patológicos (DSM5) y el bienestar psicológico". 
general terms, high scores on HFM factors are associated with health indicators, and conversely, low scores on those factors are associated with disease indicators. These results contribute to the construct validity of the HFM.

Keywords: personality traits, well-being, psychopathology, personality disorders, medical diagnosis, test validity.

Durante miles de años han sido estudiadas las características positivas de los seres humanos. Tanto las religiones como las tradiciones filosóficas de Oriente y Occidente han considerado a ese tópico como objeto de preciado interés. Confucio, Lao-Tse, Buda, Platón, Aristóteles, Moisés, Tomás de Aquino, y Al Farabi, entre otros, han intentado describir las virtudes que hacen a la excelencia moral de las personas (Cosentino, 2010). Desde comienzos del siglo XX el tema se tornó objeto de interés de la Psicologia, y ha sido considerado por los trabajos de Thorndike, Hartshorne, May, Erikson, Kohlberg, Maslow, y Jahoda, entre otros.

Los enfoques empíricos relacionados con el estudio de las características humanas positivas pueden ser clasificados en dos tipos (Chow, 2002): (a) guiados por las teorías (theory-driven), o (b) guiados por los datos (data-driven).

Los enfoques guiados por la teoría se caracterizan por diseñar a priori un modelo teórico que posteriormente se intentará corroborar empíricamente. Un ejemplo de este enfoque es la clasificación de virtudes y fortalezas de Peterson y Seligman (2004). Así, Dahlsgaard, Peterson, y Seligman (2005) propusieron que 6 virtudes estaban implícita o explícitamente mencionadas en algunos textos tradicionales filosóficos y religiosos de Oriente (e. g., hinduismo) y Occidente (e. g., filosofia ateniense). Posteriormente, análisis y debates académicos llevaron a los autores a proponer un listado de 24 fortalezas del carácter que se correspondian con esas virtudes. Esta clasificación de 6 virtudes y 24 fortalezas ha sido de uso extendido por los autores de la Psicología Positiva.

Los enfoques guiados por los datos o data-driven, se caracterizan por ser estudios inductivos que analizan datos empíricos y tienen como objetivo identificar agrupaciones de elementos (p. ej., características positivas) con el propósito de encontrar una generalización aceptable de los datos, con el fin potencial de conseguir su replicación con diferentes poblaciones.

Un estudio pionero del enfoque data-driven sobre las características positivas fue el realizado por Walker y Pitts (1998). Estos autores tuvieron por objetivo analizar las concepciones naturalisticas sobre la excelencia moral por medio de solicitar a los participantes que identifiquen los atributos de una persona de elevada moral. Posteriormente, unos jueces generaron un conjunto de descriptores morales. El resultado de este estudio fue el hallazgo de 6 clusters de atributos de características morales que llamaron basado en principios-idealista, confiable-leal, integro, bondadoso, justo, y seguro.

En otro estudio, Cawley, Martin, y Johnson (2000) partieron de la identificación de 140 palabras del diccionario que se correspondian con las virtudes morales. Estas virtudes (vinculadas a sustantivos/adjetivos) fueron transformadas en un cuestionario de autoinforme que posteriormente completaron estudiantes universitarios. Como resultado, los autores propusieron un modelo 4 dimensiones de virtudes compuesto por empatía, orden, ingenioso, y sereno. 
De Raad y van Oudenhoven (2011), utilizando una aproximación psicoléxica, les solicitaron a jueces (estudiantes y psicólogos) que identifiquen términos descriptores de virtudes de una lista de términos de diccionario que describian rasgos de las personas. Posteriormente, los participantes se organizaron en parejas, y cada par de la dupla se autoevaluó a sí mismo y a su par en cada una de las virtudes propuestas. Como resultado de este procedimiento surgió un modelo 6 factores de virtudes que estaba constituido por sociabilidad, logro, respeto, vigor, altruismo, y prudencia.

Otro estudio desde la perspectiva data-driven fue el realizado por Morales-Vives, De Raad y Vigil-Colet (2014). Los autores realizaron un proceso de varios pasos que finalizó con la identificación de 209 descriptores de virtudes. Posteriormente, un grupo de estudiantes universitarios se autoevaluó en cada uno de estos descriptores de virtudes. Como resultado, se propuso un modelo de 7 factores: autoconfianza, reflexión, serenidad, rectitud, perseverancia y esfuerzo, compasión y sociabilidad.

Finalmente, Cosentino y Castro Solano (2017), utilizando una aproximación psicoléxica, partieron de la identificación de las características humanas positivas desde el punto de vista de las personas comunes. Su investigación tuvo por objetivo desarrollar un modelo de factores de rasgos positivos humanos socialmente compartidos que pudiese ser replicable en otras poblaciones. Hasta el momento de esa investigación, los estudios sobre características positivas se habian focalizado solamente en un subtipo de rasgos positivos: los rasgos morales (e.g., virtudes y fortalezas del carácter), en tanto que talentos y habilidades habian sido sistemáticamente excluidos. Sin embargo, Cosentino y Castro Solano consideraron las características psicológicas positivas en un sentido amplio, incluyendo características sin connotación moral (e.g., tranquilidad) como así también las relacionadas con la performance (e.g., inteligencia). Su investigación tuvo por resultado el desarrollo de un modelo replicable de cinco factores denominado Modelo de los Cinco Altos o High Five Model (HFM). Los cinco factores positivos del HFM son erudición, paz, jovialidad, honestidad y tenacidad. Como rasgos positivos de la personalidad, los factores altos del HFM están presentes en cada individuo de forma relativamente estable, y están representados por características psicológicas positivas. Los factores altos presentan ciertas propiedades: pueden medirse, varian entre individuos, y supuestamente podrian ser incrementados o reducidos por influencias internas y/o externas. Estos factores del HFM son diferentes de los factores del modelo de los Cinco Grandes de la personalidad, aunque están relacionados con ellos. En este sentido, el HFM incrementa la predicción del bienestar (hedónico y eudamónico) por sobre la del modelo de los Cinco Grandes.

El estudio de Cosentino y Castro Solano (2017) representa un abordaje único y original porque, a diferencia de los estudios previamente citados, incorpora un enfoque profundamente psicoléxico (se partió de las palabras que piensan y utilizan las personas comunes, sin mediación de diccionarios), y se basó exclusivamente en procedimientos estadísticos (e.g., frecuencia de las palabras) para el análisis de los descriptores de los rasgos positivos, excluyendo la subjetividad de los juicios de expertos y jueces para clasificar y eliminar elementos en distintas instancias del proceso de investigación como es práctica habitual en este tipo de estudios. Esta aproximación conlleva a una mejor representación de los rasgos positivos internalizados por las personas en comparación con otros procedimientos. 
Si bien el estudio de Cosentino y Castro Solano (2017) sustenta no solo la relación del HFM con variables positivas, sino también la validez incremental del HFM por sobre las variables clásicas de personalidad para la predicción del bienestar, en la presente investigación se estudia la asociación del HFM con indicadores de funcionamiento óptimo y patológico. La hipótesis de este trabajo es que el HFM predice ambos extremos del continuo saludenfermedad, tanto del funcionamiento positivo como del patológico.

Por lo tanto, los objetivos del presente estudio son: a) estudiar la asociación de los factores del HFM (rasgos positivos) y el funcionamiento óptimo; b) estudiar la asociación de los factores del HFM y el funcionamiento patológico (trastornos de personalidad y síntomas psicopatológicos).

\section{MÉTODO}

\section{Participantes}

Participaron 1032 sujetos (512 hombres, 49.6\% y 519 mujeres, 50.3\%) que tenian en promedio 39.42 años $(D E=14.33)$ residentes en la Ciudad Autónoma de Buenos Aires $(n=702,68 \%)$ y el Conurbano Bonaerense $(n=$ $330,32 \%)$. La mayoría de los participantes trabajaban ( $n=822,79.7 \%)$, ubicándose en las categorías de empleado ( $n=518,50.19 \%$ ), un bajo porcentaje o bien trabajaba por su cuenta $(n=221,21 \%)$ o se ubicaba en la categoría de patrón o empleador $(n=73,7 \%)$. En esta muestra, 11 participantes manifestaron trabajar $(1.51 \%)$ pero no percibian salario por ello.

En cuanto al nivel de estudios, la mayoria de los sujetos $(63 \%, n=650)$ refirió tener estudios universitarios o terciarios completos. El 40\% restante había completado la educación secundaria $(n=382)$. La mayoría de los participantes pertenecían a un nivel socioeconómico medio $(n=712 ; 69 \%)$ y medio-alto ( $n=320 ; 24 \%)$.

Se trata, por lo tanto, de una muestra de conveniencia, de población general urbana altamente educada y económicamente activa, de nivel socioeconómico medio a medio-alto.

La participación fue voluntaria, consentida, y anónima; y no se dio contribución económica alguna por participar. Para este estudio se utilizaron los datos de población general no consultante. Los participantes fueron reclutados por estudiantes avanzados de la carrera de Psicología que estaban realizando sus prácticas de investigación al finalizar sus estudios de licenciatura.

\section{Instrumentos}

High Five Inventory (HFI; Cosentino \& Castro Solano, 2017). El HFI es un instrumento de medición de los factores del HFM, llamados factores altos o altos (Cosentino y Castro Solano (2017). Los factores altos del HFM que mide el HFI son: erudición, paz, jovialidad, honestidad y tenacidad. Este instrumento fue obtenido a través de un procedimiento inductivo que partió del punto de vista de las personas comunes sobre las características positivas (morales o no morales). La escala está compuesta por 23 ítems. Se le pide al participante que responda a los ítems (p. ej., "Tengo paciencia") utilizando una escala que va de 1 (nunca) a 7 (siempre). A mayor puntuación de cada subescala, más elevado factor alto. El HFI presentó las siguientes valideces: convergente y divergente con relación a la clasificación Values in Action de 
Peterson y Seligman (2004), e incremental por sobre los factores y facetas del Modelo de los Cinco Grandes en la predicción de las tres dimensiones del modelo Salud Mental Positiva. Además, el HFI presentó un buen ajuste a los datos tanto de la muestra de generación (p. ej., $C F I=0.968$ ) como de la muestra de confirmación (p. ej., $C F I=0.963$ ). La confiabilidad alfa y omega para cada factor es superior a .80. La consistencia interna de los factores se muestra en la tabla 1.

Symptom Checklist-90-R (SCL-90-R; Casullo, 1998; Derogatis, 1983). E1 SCL-90-R evalúa sintomatología específica experimentada durante la última semana en nueve dimensiones (somatización, obsesiones y compulsiones, sensitividad interpersonal, depresión, ansiedad, hostilidad, ansiedad fóbica, ideación paranoide y psicoticismo). Asimismo, se incluyen nueve ítems críticos $\mathrm{y}$ tres indices (indice de severidad global, indice positivo de malestar, y total de síntomas positivos). El SCL-90-R está compuesto por 90 items que se responden en una escala de formato Likert en 5 puntos que va de 1 (nada) a 5 (mucho). Los estudios argentinos demostraron las propiedades psicométricas del instrumento (Casullo \& Castro Solano, 1999). Para este estudio se utilizó el índice de severidad global como indicador general de sintomatología.

Personality Inventory for DSM5 (PID-5-BF). Se utilizó la versión abreviada de 220 items del PID5 Inventory (Krueger, Derringer, Markn, Watson \& Skodol, 2012), adaptada en Argentina por Fernández Liporace y Castro Solano (2015). El PID-5-BF (Krueger, Derringer, Markn, Watson \& Skodol, 2013) se compone de 25 ítems que evalúan los cinco rasgos de personalidad disfuncionales dominantes propuestos en la sección III de la quinta versión del Manual Diagnóstico y Estadístico de los Trastornos Mentales -DSM-5 (American Psychiatric Association, 2013): afectividad negativa, desapego, antagonismo, desinhibición y psicoticismo. El PID-5-BF utiliza un formato de respuesta Likert de 4 puntos que va de 0 (muy falso o a menudo falso) a 3 (muy cierto o a menudo verdadero). Los estudios argentinos corroboran la estructura de cinco factores originariamente propuesta por el modelo y la relación con el modelo de los rasgos de personalidad normal del modelo de los cinco grandes. La fiabilidad de cada una de las escalas se encuentra dentro del rango .86 a .90 (Góngora \& Castro Solano, 2017). Para este estudio se utilizó una puntuación global, que tenía una fiabilidad de .87

Mental Health Continuum- Short Form (MHC-SF). Es una escala compuesta de 14 items (Keyes, 2005) que evalúa el bienestar emocional (3 items), psicológico (6 items) y social (5 items). La escala tiene un formato de respuesta Likert con 5 opciones de respuesta. Se responde con relación a cómo el participante se ha sentido en el último mes, que va de 0 (nunca) a 5 (todos los dias). El MHC-SF ha demostrado tener una buena consistencia interna $(>.70)$ y presenta una adecuada validez discriminante (estructura de tres factores, bienestar emocional, personal y social) en estudios realizados con población adulta en los Estados Unidos, China, Italia, Irán, Canadá, Polonia y Sudáfrica (Gallagher, Lopez, \& Preacher, 2009; Gilmour, 2014; Joshanloo, Wissing, Khumalo, \& Lamers, 2013; Karas, et al., 2014; Keyes, 2005; Lamers, Westerhof, Bohlmeijer, ten Klooster, \& Keyes, 2011; Petrillo, et al., 2015; Robitschek \& Keyes, 2009; Yin, et al., 2013). Para este estudio se utilizó la versión argentina. El estudio realizado en población local confirmó las propiedades psicométricas del instrumento (adecuada confiabilidad, estructura factorial y validez convergente; Lupano Perugini, de la Iglesia, Castro Solano, \& Keyes, 2016). En la muestra del presente estudio la 
consistencia interna del instrumento fue de alfa de Cronbach $=.86$ para bienestar emocional, alfa de Cronbach $=.86$ para bienestar personal y alfa de Cronbach $=.75$ para bienestar social.

Encuesta de Factores de Riesgo para Enfermedades No Trasmisibles (Ministerio de Salud de la Nación Argentina, 2011). Esta encuesta sigue los lineamientos de la Organización Mundial de la Salud (World Health Organization, 2005) para la detección de factores de riesgo para enfermedades crónicas en personas mayores de 18 años. Consta de 97 preguntas organizadas en módulos sobre: percepción de la salud en general, actividad física, hipertensión arterial, peso corporal, alimentación, colesterol, consumo de alcohol, consumo de tabaco, diabetes y prácticas de salud preventivas. La encuesta ha sido adecuada a la población argentina y testeada en todo el país como parte de estudios nacionales sobre factores de riesgo. Se evalúan los 8 factores de riesgo presentes en cada persona. Según los lineamientos de la OMS, la presencia de más de un factor aumenta el riesgo de morbilidad y mortalidad. Para el presente trabajo la presencia de un factor o ninguno, se caracterizó como perfil de bajo riesgo. La presencia de dos factores es categorizada como riesgo medio y la presencia de 3 o más factores es caracterizada como perfil de alto riesgo.

\section{Procedimiento}

Los protocolos fueron administrados por estudiantes avanzados de la Carrera de Psicología de una universidad situada en la Ciudad Autónoma de Buenos Aires, los cuales se encontraban realizando sus prácticas profesionales en el área de investigaciones. Tenian como instrucciones administrar la batería de pruebas a población general no consultante residente en la Ciudad de Buenos Aires y sus alrededores. Los encuestados respondieron individualmente a los instrumentos.

No se incluyeron protocolos con datos ausentes. En función de esto se eliminaron 12 protocolos. Los datos fueron analizados con el paquete estadístico SPSS versión 17.

Para analizar los datos se generaron clasificaciones de los valores de algunas variables que fuesen acordes con los análisis estadísticos a utilizar en esta investigación. Consistentemente, se creó una nueva variable basada en las puntuaciones del MHC-SC del modelo de Salud Mental Positiva, que constó de tres categorias ordenadas: flourishing, salud mental moderada y languishing. Además, se generó otra variable discreta ordenada basada en la puntuación en factores de riesgo para la salud según los criterios de la OMS: sin riesgo, riesgo medio y riesgo alto. También, se discretizaron los factores altos del HFM en las categorias alto vs. bajo, los rasgos de personalidad patológica en las categorias presencia y ausencia, y sintomas psicológicos en presencia y ausencia. Con los datos de las nuevas variables se llevaron a cabo dos análisis de correspondencias múltiples. El primero establecía la relación entre los altos del HFM y las variables positivas (alto flourishing y sin riesgo para la salud) y el segundo, que establecía la relación entre los altos y funcionamiento patológicos (rasgos patológicos de la personalidad y presencia de sintomas psicopatológicos). 


\section{RESULTADOS}

\section{Estadísticos Descriptivos}

Respecto de la nueva variable derivada de las puntuaciones del MHCSC, basada en la clasificación de Salud Mental Positiva propuesta por Keyes (2005), se consideró que el participante evaluado tenía una salud mental óptima (i.e., flourishing) si reportaba haber experimentado todos los dias o casi todos los días del último mes al menos 1 de los 3 indicadores de bienestar hedónico y 6 de los 11 indicadores de bienestar eudaimónico (i. e., funcionamiento positivo). Se ubicaron en esta categoria el $44 \%$ de los participantes. Aquellos que reportaron que durante el último mes presentaron bajos niveles (e.g., nunca, o una o dos veces al mes) en al menos un indicador de bienestar hedónico, y bajos niveles en al menos 6 indicadores de bienestar eudoimónico se consideraron que presentaban salud mental baja (languishing). Se ubicaron en esta categoria apenas el 3\% de los participantes evaluados. Aquellos que no se ubican ni en flourishing ni en languishing se clasificaron en salud mental moderada. Se ubicaron en esta categoría el 53\% de los participantes.

Se siguió un procedimiento similar al presentado arriba, a fin de establecer la presencia de factores de riesgo para la salud, según evalúa la Encuesta de Factores de Riesgo para Enfermedades No Trasmisibles. Los participantes que presentaron uno o ningún factor de riesgo fueron considerados sin riesgo. En esta categoría se ubicaron el $41 \%$ de los participantes. La presencia de dos factores se consideró riesgo medio $131 \%$ de los participantes) y la presencia de 3 o más factores se caracterizó como alto riesgo. En esta última categoría se ubicaron el $28 \%$ de los encuestados.

Respecto de la variable trastornos psicológicos se seleccionó el índice global de severidad del SCL-90-R como indicador de sintomatología clínica. Se consideró a una puntuación mayor al percentil 75 en ese índice como potencial caso clínico. Utilizando este criterio, se consideró que se presentaba alguna problemática psicológica en el $44 \%$ de la muestra y ningún síntoma psicológico al 56\% restante.

En función de los datos analizados puede caracterizarse la muestra estudiada como de buena salud mental y de bajo riesgo. Seguidamente, se presentan los estadísticos descriptivos del Modelo de los Cinco Altos y otras variables analizadas en este estudio.

Tabla 1

Estadísticos descriptivos de los factores altos, rasgos patológicos de la personalidad y síntomas psicológicos.

\begin{tabular}{lccc}
\hline & $M$ & $D E$ & Alfa de Cronbach \\
\cline { 2 - 4 } HFI Erudición & 5.96 & 0.90 & .84 \\
HFI Paz & 5.32 & 1.28 & .86 \\
HFI Jovialidad & 5.48 & 1.20 & .88 \\
HFI Honestidad & 6.42 & 0.70 & .88 \\
HFI Tenacidad & 6.34 & 0.80 & .86 \\
Rasgos Psicopatológicos (PID-5) & 19.02 & 10.87 & .87 \\
Síntomas (SCL-90 -R - Ind G. Severidad) & 0.15 & 0.04 & - \\
\hline
\end{tabular}

Como puede verse en la tabla 1, la mayoría de los participantes obtuvieron altas puntuaciones en todos los marcadores del HFM, siendo la 
puntuación más alta para honestidad y la más baja para paz. En cuanto a los rasgos patológicos de personalidad, los participantes podian obtener hasta un total de 75 puntos (alta presencia de rasgos patológicos en todos los items) siendo la media de 19.02 obtenida por los participantes. Por lo tanto, se trata de una población que mayoritariamente presenta bajas puntuaciones en rasgos patológicos de la personalidad.

\section{Relaciones entre HFM y funcionamiento óptimo}

Para establecer las asociaciones entre rasgos positivos (los factores altos del HFM) y funcionamiento óptimo, se llevó a cabo un análisis de correspondencias múltiples. Dado que dos de las tres variables consideradas se habian discretizado (i.e., factores de riesgo y Salud Mental) se discretizaron también cada uno de los factores del HFM en los valores alto y bajo, tomando en cuenta la media en cada una de las dimensiones (valor alto = por encima de la media; valor bajo = por debajo de la media). En el análisis se incluyeron los tres modelos comentados (HFM, factores de riesgo y salud mental positiva). E1 análisis permitió aislar dos dimensiones que explicaban el 46\% de la varianza. La primera de ellas explicaba un porcentaje importante de la varianza total $(30 \%)$. Esta dimensión describe un continuo que va de alta presencia de rasgos positivos a baja presencia. En un extremo si situarian aquellas personas con los factores del HFM elevados y, en el otro extremo, las personas de factores del HFM bajos. La segunda dimensión explicaba el 16\% restante. Se trata de un continuo que va del alto riesgo para la salud y baja salud psicológica (languishing) a un perfil de óptimo funcionamiento psicológico y sin riesgo para la salud. El análisis del gráfico de correspondencias permite identificar dos agrupaciones: 1) La alta jovialidad y alta paz asociadas con la alta percepción de bienestar hedónico y eudaimónico (flourishing) y con la ausencia de factores de riesgo para la salud; 2) baja jovialidad y baja paz asociadas con salud mental moderada y pobre (languishing; ver figura 1).

\section{Relaciones entre HFM y funcionamiento psicológico deficiente}

Un procedimiento similar al anterior se siguió para establecer la relación entre el HFM y variables vinculadas con la psicopatología. Se incluyeron en el análisis de correspondencias múltiples los factores del HFM categorizados en alto y bajo. Asimismo, se incluyó la presencia/ausencia de sintomatología psicológica (puntuación derivada del SCL-90-R, Indicador GSI) y la presencia/ausencia de rasgos patológicos de personalidad. Para esta última se construyó una variable nominal que resultó de la puntuación global de la escala PID-5-BR, se asignó la categoría de presencia de rasgos patológicos de la personalidad a aquellas puntuaciones globales del PID-5-BR que superaban el percentil 75 y ausencia de rasgos patológicos de la personalidad a aquellas inferiores al percentil 25. En este estudio se utilizó un criterio empírico de corte, debido a que estudios previos (Samuel, 2013) indicaron que las diferencias entre utilizar un criterio de corte racional y un criterio empírico en las frecuencias de diagnóstico eran mínimas, porque ambos métodos eran equivalentes. El análisis de correspondencias múltiples permitió aislar dos dimensiones que explicaban el $52 \%$ de la varianza. La primera de ellas explicaba un porcentaje significativo de la varianza total (31\%). Esta dimensión describe un continuo que va de altos a bajos valores en los factores del HFM. Así, en un extremo se ubican las personas que presentan elevados niveles de los factores del HFM y en el otro extremo se 
sitúan las de bajo nivel en los factores del HFM. La segunda dimensión explicaba el $21 \%$ restante. Se trata de un continuo que va de ausencia a presencia de sintomas y de rasgos de personalidad patológicos. El análisis del gráfico de correspondencias permite realizar dos agrupaciones: Por un lado, los altos valores del HFM asociados a ausencias de sintomas psicopatológicos y de rasgos de personalidad patológicos; por el otro, bajos valores en los factores del HFM asociados con la presencia de sintomas y rasgos de personalidad psicopatológicos (ver figura 2).

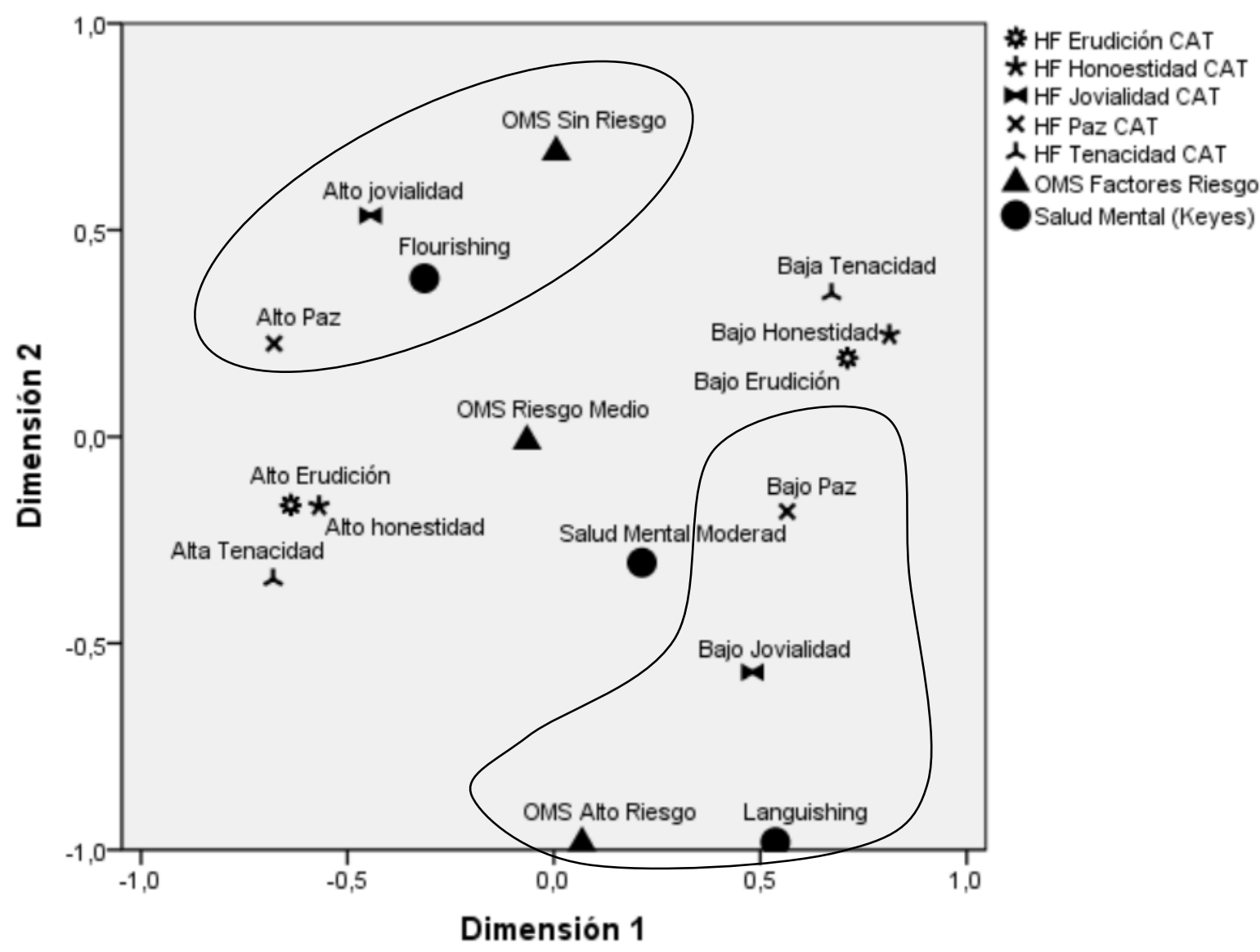

Figura 1. High Five Model y Funcionamiento óptimo 


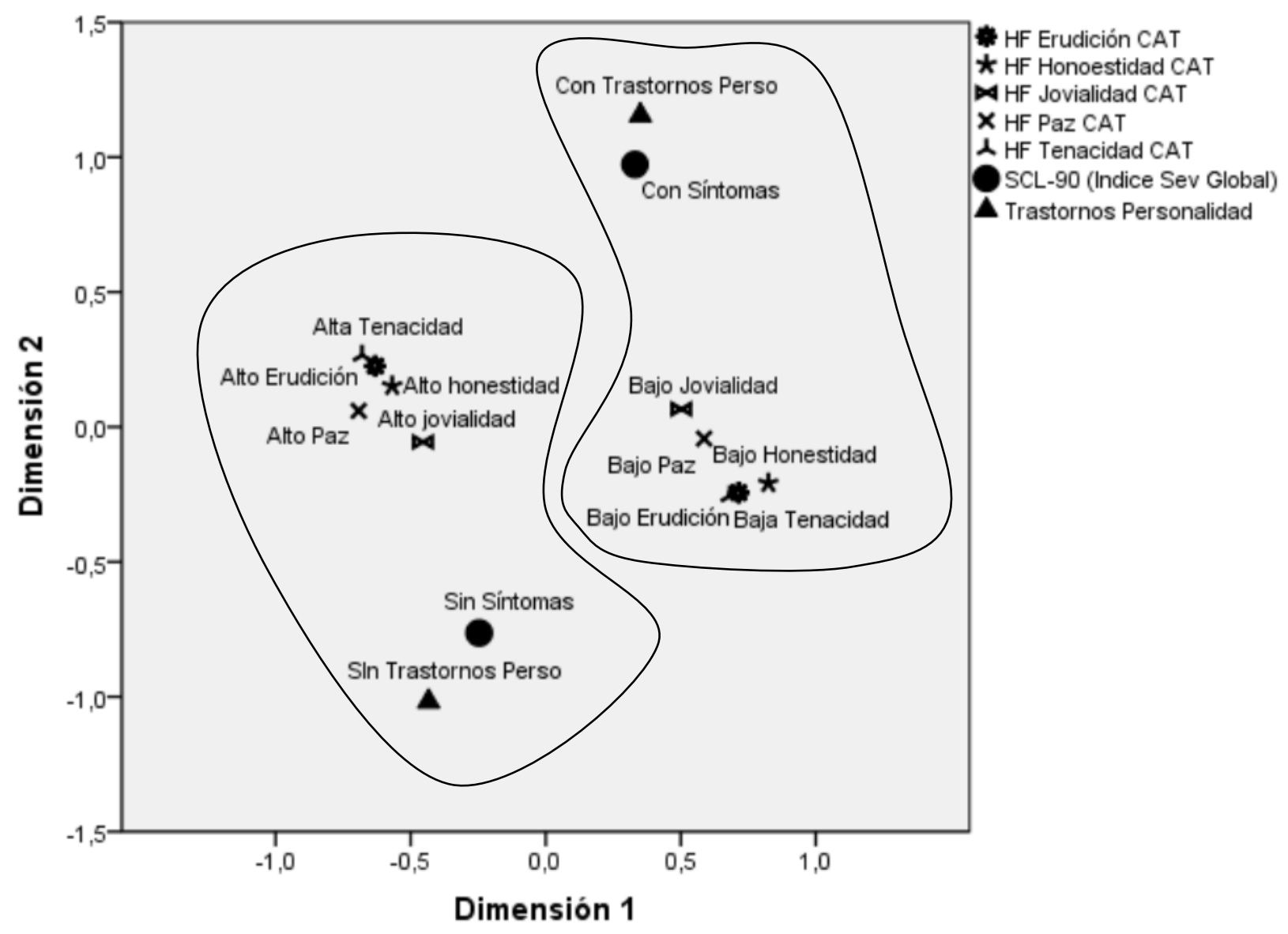

Figura 2. High Five Model y Funcionamiento psicológico deficiente.

\section{DISCUSIÓN}

Consideramos al HFM como un modelo de rasgos positivos de la personalidad, de amplio potencial, derivado de modo inductivo de las ideas que tienen las personas comunes acerca de las características psicológicas más valoradas. Hasta ahora, los estudios realizados con el HFM habian permitido confirmar la replicabilidad de estructura factorial en población adulta, y que permite predecir la salud mental positiva. Asimismo, esos estudios confirmaron la validez incremental del HFM por sobre los factores y facetas de personalidad del modelo de los Cinco Grandes para la predicción de diferentes tipos de bienestar de la Salud Mental Positiva, lo que constituye un indicador adicional de la validez de constructo del HFM (Cosentino \& Castro Solano, 2017).

En este estudio se demostró que elevados factores altos del HFM no solo permiten establecer una separación de las variables psicopatológicas, sino que están asociados significativamente con las variables positivas (alto flourishing y pocos o ningún factor de riesgo para la salud). En efecto, los altos factores altos del HFM, especialmente alta paz y alta jovialidad, están asociados con una mejor salud mental positiva y bajo riesgo de enfermedad médica. El factor paz puede entenderse como un factor relacionado con la tolerancia con los demás y la tranquilidad mental característica de aquellas personas que 
registran bajas preocupaciones personales con la vida. El factor jovialidad está relacionado con el sentido del humor y la simpatía y la diversión, por lo que podría considerarse como un indicador de un estado de ánimo positivo.

Estudios anteriores relacionados con otras variables positivas están en relación con lo hallado en este estudio. Las investigaciones más modernas sobre factores disposicionales de la personalidad (e.g., fortalezas y virtudes) indican que este tipo de constructos resultan predictores importantes de funcionamiento positivo tales como afecto positivo (Drozd, Mork, Nielsen, Raeder, Bjorkli, 2014; Gander, Proyer, Ruch, \& Wyss, 2013), bienestar psicológico (Duan, Ho, Tang, Li, \& Zhang, 2013; Mongrain, \& AnselmoMatthews, 2012; Young, Kashdan, \& Macatee, 2015; Wood, Linley, Matlby, Kashdan, \& Hurling, 2011) o el buen rendimiento en el trabajo (Elston, \& Boniwell, 2011; Meyers \& Van Woerkom, 2014).

El principal aporte de esta investigación es otorgar validez externa adicional al HFM. El HFM permite predecir de forma relativamente adecuada ambos extremos del continuo salud-enfermedad. Elevados factores altos se presentaron asociados con un funcionamiento óptimo y bajos factores altos se presentaron asociados con un funcionamiento relacionado, en última instancia, a la patología. Si bien la idea de continuidad sindrómica no es nueva, muy pocos modelos psicológicos han vinculado la salud y la enfermedad de forma conjunta (e.g., Leary, 1957; Millon, 1996; Millon \& Everly, 1994; Offer \& Sabshin, 1991; Strack \& Lorr, 1994). El modelo de personalidad de Millon y el abordaje del continuo salud-enfermedad del modelo de Keyes son dos importantes excepciones. Si bien con el correr de los años se ha incrementado el reconocimiento de que las concepciones sobre normalidad y patología deberian estar organizadas en un único marco conceptual, a la fecha no contamos con una clasificación integrada de psicopatología y normalidad, de amplia aceptación en la comunidad científica. Los modelos que tratan el tópico resultan más la excepción que la regla.

Para el campo aplicado, la evaluación de los rasgos positivos derivados de una modelo psicoléxico resulta una variante breve que permite efectuar la predicción tanto del funcionamiento positivo como del patológico porque el HFI es un instrumento sencillo y de fácil administración que demora no más de 5 minutos. Resulta una alternativa más moderna y eficiente frente a la utilización de los inventarios clásicos de personalidad y otras técnicas de evaluación que tienen una elevada cantidad de ítems, de evaluación mucho más compleja, y que requieren, en muchos casos, de softwares especializados para su evaluación y corrección.

\section{Limitaciones y perspectivas futuras}

Una de las limitaciones de este estudio es haber utilizado categorización de variables cuantitativas. Este tipo de procedimiento reduce la información original de las variables. Estudios futuros podrian utilizar estas mismas variables para realizar análisis más específicos con el fin de generar una predicción más exacta de modelo investigado sobre las variables criterio.

\section{REFERENCIAS}

American Psychiatric Association (2013). Diagnostic and statistical manual of mental disorders, (DSM-5®). Arlington, VA: American Psychiatric Association. https://doi.org/10.1176/appi.books.9780890425596 Cosentino, A. C. (2010). Las fortalezas del carácter. En A. Castro Solano (Ed.), Fundamentos de Psicología Positiva (pp. 111-135). Buenos Aires, Argentina: Paidós. 
Cosentino, A. C., \& Castro Solano, A. (2017). The High Five: Associations of the five positive factors with the Big Five and well-being. Frontiers in Psychology, 8, 1250. https://doi.org/10.3389/fpsyg.2017.01250

Casullo, M. M. (1998). El Listado de Sintomas SCL-90-R de Derogatis [The Derogatis' Symptom Checklist90-R] . Buenos Aires, Argentina : Departamento de Publicaciones, Facultad de Psicologia, Universidad de Buenos Aires

Casullo, M. \& Castro Solano, A. (1999). Síntomas psicopatológicos en estudiantes adolescentes argentinos. Aportaciones del SCL-90-R. Anuario de Investigaciones, Facultad de Psicologí, Universidad de Buenos Aires, 7, 147-157.

Cawley III, M. J., Martin, J. E., \& Johnson, J. A. (2000). A virtues approach to personality. Personality and Individual Differences, 28(5), 997-1013. https://doi.org/10.1016/S0191-8869(99)00207-X

Chow, S. L. (2002). Methods in psychological research. Encyclopedia of Life Support Systems. Oxford, UK: EOLSS Publishers.

Dahlsgaard, K., Peterson, C., \& Seligman, M. E. P. (2005). Shared virtue: The convergence of valued human strengths across culture and history. Review of General Psychology, 9(3), $203-213$. https: / / doi.org/10.1037/1089-2680.9.3.203

De Raad, B. \& van Oudenhoven, J. P. (2011). A psycholexical study of virtues in the Dutch language, and relations between virtues and personality. European Journal of Personality, 25(1), 43-52. https://doi.org/10.1002/per.777

Derogatis, L. R. (1983) Symptom Checklist-90-Revised. San Antonio, TX : Pearson.

Drozd, F., Mork, L., Nielsen, B., Raeder, S., \& Bjørkli, C. A. (2014). Better Days-A randomized controlled trial of an internet-based positive psychology intervention. The Journal of Positive Psychology, 9(5), 377-388. https://doi.org/10.1080/17439760.2014.910822

Duan, W., Ho, S. M. Y., Yu, B., Tang, X., Zhang, Y., Li, T., \& Yuen, T. (2012). Factor structure of the Chinese virtues questionnaire. Research on Social Work Practice, 22(6), 680-688. https: / / doi.org/10.1177/1049731512450074

Elston, F. \& Boniwell, I. (2011). A grounded theory study of the value derived by women in financial services through a coaching intervention to help them identify their strengths and practice using them in the workplace. International Coaching Psychology Review, 6(1), 16-32.

Fernández Liporace, M. L. \& Castro Solano, A. (2015). Personality Inventory for DSM5. Versión Argentina. Forma Adulta. Manuscrito no publicado.

Gallagher, M. W., Lopez, S. J., \& Preacher, K. J. (2009). The hierarchical structure of well-being. Journal of Personality, 77(4), 1025-1050. https://doi.org/10.1111/j.1467-6494.2009.00573.x

Gander, F., Proyer, R. T., Ruch, W., \& Wyss, T. (2013). Strength-based positive interventions: Further evidence for their potential in enhancing well-being. Journal of Happiness Studies, 14(4) 1241-159. https: / /doi.org/10.1007/s10902-012-9380-0

Gilmour, H. (2014). Positive mental health and mental illness. Health Reports, 25, 3-9.

Gongora, V. \& Castro Solano, A. (2017, en prensa). Pathological personality traits (DSM-5), risk factors and mental health. Sage Open.

Joshanloo, M., Wissing, M. P., Khumalo, I. P., \& Lamers, S. M. A. (2013). Measurement invariance of the Mental Health Continuum-Short Form (MHC-SF) across three cultural groups. Personality and Individual Differences, 55(7), 755-759. https://doi.org/10.1016/j.paid.2013.06.002

Karaś, D., Cieciuch, J., \& Keyes, C. L. M. (2014). The Polish adaptation of the Mental Health ContinuumShort Form (MHC-SF). Personality and Individual Differences, 69, $104-109$. https://doi.org/10.1016/j.paid.2014.05.011

Keyes, C. L. M. (2005). Mental illness and/or mental health? Investigating axioms of the complete state model of health. Journal of consulting and clinical psychology, 73(3), 539-548. https: / / doi.org/10.1037/0022-006X.73.3.539

Krueger, R. F., Derringer, J., Markon, K. E., Watson, D., \& Skodol, A. E. (2012). Initial construction of a maladaptive personality trait model and inventory for DSM-5. Psychological Medicine, 42(09), 18791890. https://doi.org/10.1017/S0033291711002674

Krueger, R. F., Derringer, J., Markon, K., Watson, D., \& Skodol, A. (2013). The personality inventory for DSM-5-brief form (PID-5-BF)-adult. American Psychiatric Association. Retrieved from Retrieved from http://www.psychiatry.org/practice/dsm/dsm5/online-assessment-measures

Lamers, S. M. A., Westerhof, G. J., Bohlmeijer, E. T., ten Klooster, P. M., \& Keyes, C. L. M. (2011). Evaluating the psychometric properties of the Mental Health Continuum-Short Form (MHC-SF). Journal of Clinical Psychology, 67(1), 99-110. https://doi.org/10.1002/jclp.20741

Leary, T. (1957). Interpersonal diagnosis of personality. New York: Ronald Press.

Lupano Perugini, M. L., de la Iglesia, G., Castro Solano, A., \& Keyes, C. L. M. (2017). The Mental Health Continuum-Short Form (MHC-SF) in the Argentinean Context: Confirmatory Factor Analysis and Measurement Invariance. Europe's Journal of Psychology, 13(1), 93-108. https://doi.org/10.5964/ejop.v13i1.1163

Meyers, M. C. \& van Woerkom, M. (2014). Effects of a Strengths Intervention on Work Engagement and Burnout: Positive Affect as a Mediator. Academy of Management Proceedings, 2014(1), 15558-15563. https: / /doi.org/10.5465/AMBPP.2014.15558abstract

Millon, T. (1996). Disorders of Personality. New York: Wiley \& Sons

Millon, T. \& Everly, G. (1994). La personalidad y sus trastornos. Barcelona: Martícez Roca.

Ministerio de Salud de la Nación Argentina. (2011). Segunda encuesta nacional de factores de riesgo para enfermedades no transmisibles. Primera Edición. Buenos Aires: Ministerio de Salud de la Nación Argentina

Mongrain, M. \& Anselmo-Matthews, T. (2012). Do positive psychology exercises work? A replication of Seligman et al. Journal of Clinical Psychology, 68(4), 382-389. https://doi.org/10.1002/jclp.21839 
Morales-Vives, F., De Raad, B., \& Vigil-Colet, A. (2014). Psycho-Lexically Based Virtue Factors in Spain and Their Relation With Personality Traits. The Journal of General Psychology, 141(4), 297-325. https://doi.org/10.1080/00221309.2014.938719

Offer, D. \& Sabshin M. (1991). The diversity of normal behavior. New York: Basic Books

Peterson, C. \& Seligman, M. E. P. (2004). Character strengths and virtues: A handbook and classification. New York, NY, US: Oxford University Press

Petrillo, G., Capone, V., Caso, D., \& Keyes, C. L. M. (2015). The Mental Health Continuum-Short Form (MHC-SF) as a measure of well-Being in the Italian context. Social Indicators Research, 121(1), 291312. https://doi.org/10.1007/s11205-014-0629-3

Robitschek, C., \& Keyes, C. L. M. (2009). Keyes's model of mental health with personal growth initiative as a parsimonious predictor. Journal of Counseling Psychology, 56(2), 321-329. https://doi.org/10.1037/a0013954

Strack, S., \& Lorr, M. (1994). Differentiating normal and abnormal personalities. New York, NY: Springer

Walker, L. J., \& Pitts, R. C. (1998). Naturalistic conceptions of moral maturity. Developmental Psychology, 34(3), 403-419. https://doi.org/10.1037/0012-1649.34.3.403

Wood, A. M., Linley, P. A., Matlby, J., Kashdan, T. B., \& Hurling, R. (2011). Using personal and psychological strengths leads to increases in well-being over time: A longitudinal study and the development of the strengths use questionnaire. Personality and Individual Differences, 50, 15-19. https://doi.org/10.1016/j.paid.2010.08.004

World Health Organization. (2005). Preventing chronic diseases: A vital investment. Geneva: World Health Organization.

Yin, K. L., He, J. M., \& Fu, Y. F. (2013). Positive mental health: measurement, prevalence, and correlates in a Chinese cultural context. En C. L. M. Keyes (Ed.), Mental Well-Being (pp. 111-132). Springer, Dordrecht. Recuperado de https://link.springer.com/chapter/10.1007/978-94-007-5195-8_6

Young, K. C., Kashdan, T. B., \& Macatee, R. (2015). Strength balance and implicit strength measurement: New considerations for research on strengths of character. The Journal of Positive Psychology, 1O(1), 17-24. Doi:10.1080/17439760.2014.92040624.

Recibido 15-08-2017 | Aceptado 18-09-2017 
Castro Solano, A. \& Cosentino, A. C. / Psicodebate, 17(2), 69 - 82.

Psicodebate, Vol. 17, No 2, Diciembre 2017 - Mayo 2018

ISSN: 1515-2251 e-ISSN: 2451-6600 